\title{
Rash in systemic lupus erythematosus: prevalence and relation to cutaneous and non-cutaneous disease manifestations
}

\author{
Arjeh J Wysenbeek, Daniele Guedj, Mirit Amit, Abraham Weinberger
}

\begin{abstract}
Rash is a common cutaneous sign in patients with systemic lupus erythematosus (SLE) but no data regarding its prevalence or relation to disease manifestations are known, possibly due to the fact that it is a common but nonspecific sign. Rash was present in 48/81 (59\%) patients with SLE. Patients with rash had more cutaneous symptoms and signs, lymphadenopathy, increased levels of antibodies to double stranded DNA and decreased complement levels. They were also receiving a higher dose of prednisone. There was no difference between patients with and without rash with respect to renal or central nervous system disease. Rash did not correlate with the disease activity index and clinical parameters of disease exacerbation.
\end{abstract}

Cutaneous manifestations are common in patients with systemic lupus erythematosus (SLE). Various forms of these manifestations have been described, such as malar rash, discoid lesions, alopecia, photosensitivity, and the typical rash of subacute cutaneous lupus erythematosus. ${ }^{1}$

Non-specific rash is also common among patients with SLE, as noted by Rothfield, who observed that 'the second most common skin finding is a nonspecific maculopapular rash resembling a drug eruption' ${ }^{2}$ Regardless of the high prevalence of this cutaneous manifestation, however, hardly any data have been reported with respect to its prevalence or relation to other disease signs. ${ }^{3}$ This might be due to the fact that although rash has a high prevalence in patients with SLE, it is a non-specific sign. Thus most research has focused on the more specific cutaneous manifestations of SLE.

In this study we decided to examine rash in patients with SLE, its prevalence, relation to other disease symptoms, signs and laboratory tests, and the relation of rash to disease activity.

\section{Patients and methods}

Included in the study were 81 patients with SLE who were followed up at the Beilinson Medical Center and diagnosed according to the American Rheumatism Association revised criteria for SLE. ${ }^{4}$ Data from each examination were entered prospectively into a computerised database. At each examination the patient's medical history was recorded and a physical examination performed. All data were immediately converted by the examining doctor into 25 medical history and 29 physical examination variables, all on a scale of $0-3$. Patients were assessed by one of three doctors from the rheumatology unit.

Rash, when assessed by the doctor, was defined on clinical grounds as the occurrence of any cutaneous eruption that was not defined, or was concurrent with various specific cutaneous manifestations such as malar rash, discoid lesions, skin vasculitic ulcers, the typical rash of subacute cutaneous lupus (papulosquamous or annular) or cutaneous vasculitis (palpable purpura, microinfarcts, livedo reticularis), which were monitored separately. Rash was also assessed by the history of the patient. For the various evaluations we used the doctor's assessment of the rash.

\section{RELATION BETWEEN RASH AND DISEASE}

MANIFESTATIONS

Patients were divided into two groups according to the presence (rash $>0$ in at least one examination) or absence ( $\mathrm{rash}=0$ in all examinations) of rash. The two groups were compared for various disease variables, which were recorded in the range $0-3$. For each patient the maximum observed value for variables was recorded, and group medians were compared for each variable.

\section{RELATION BETWEEN RASH AND DISEASE} ACTIVITY

For each patient the examination with the highest recorded value for rash was marked, and a rash rating of $0-3$ (none, mild, moderate, severe) was correlated with the disease activity index in the range $1-4$ and various clinical and laboratory test variables, ${ }^{5}$ all monitored at the same examination.

\section{RELATION BETWEEN RASH AND OTHER CUTANEOUS SIGNS}

The number of patient examinations with a positive recording for rash and a concomitant negative recording for malar rash, discoid lesions, cutaneous ulcers, and subacute cutaneous lupus, was divided by the number of patient examinations with a positive recording for rash and a concomitant positive recording for at least one of the other cutaneous signs.

\section{STATISTICAL EVALUATION}

Groups interval and ordinal data were compared by Wilcoxon's rank sum test. Nominal data 
were compared by the $\chi^{2}$ test. Correlation was by Spearman's rank correlation coefficient. Significance was not adjusted for multiple comparisons. Data are presented as mean (standard error of mean (SE)).

\section{Results}

Table 1 gives the prevalence of the various cutaneous manifestations in our patient group. As shown, photosensitivity is the cutaneous finding with the highest prevalence $(71 \%)$, followed by rash (59\%), alopecia (58\%), and malar rash $(49 \%)$.

Table 2 gives the prevalence of rash according to the medical history of the patient and examination by the doctor. These two assessments correlated significantly $(r=0.8 \mathrm{p}<0.001)$.

Forty eight patients were allocated to the group with rash and 33 patients to the group with no rash. The group with rash was significantly younger $(39 \cdot 6(1 \cdot 9) v 48 \cdot 2(2 \cdot 9)$ years $(\mathrm{p}=0.013))$. There was no significant difference in sex distribution $(85 \%$ v $93 \%$ women $)$, education level $(11.7(0.5) v 10.5(0.6)$ years $)$ or

Table 1 Prevalence of the various cutaneous signs in our group of 81 patients. All data are based on examination by a doctor except photosensitivity, which is based on the patient's medical history

\begin{tabular}{ll}
\hline Cutaneous sign & Prevalence (\%) \\
\hline Photosensitivity & 71 \\
Rash & 59 \\
Alopecia & 58 \\
Malar rash & 49 \\
Discoid lesions & 19 \\
Cutaneous vasculitis & 17 \\
Subacute cut. LE & 13 \\
Cutaneous ulcers & 8 \\
\hline
\end{tabular}

Table 2 Prevalence of rash as determined by patient's medical history and examination by a doctor. Results are the highest recorded observations for each patient

\begin{tabular}{lll}
\hline $\begin{array}{l}\text { Severity of } \\
\text { rash }\end{array}$ & $\begin{array}{l}\text { No of patients } \\
\text { (\%) by history }\end{array}$ & $\begin{array}{l}\text { No of patients } \\
\text { (\%) by doctor's } \\
\text { examination }\end{array}$ \\
\hline None & $22(27)$ & $33(41)$ \\
Mild & $12(15)$ & $11(14)$ \\
Moderate & $24(30)$ & $18(22)$ \\
Severe & $23(28)$ & $19(23)$
\end{tabular}

Table 3 Comparison of the groups with and without rash for disease manifestations and laboratory tests. Variables (mean (SE)) were recorded according to a doctor's examination, except photosensitivity which is based on the patient's medical history, in the range 0-3. Values in brackets are the prevalence of the variables

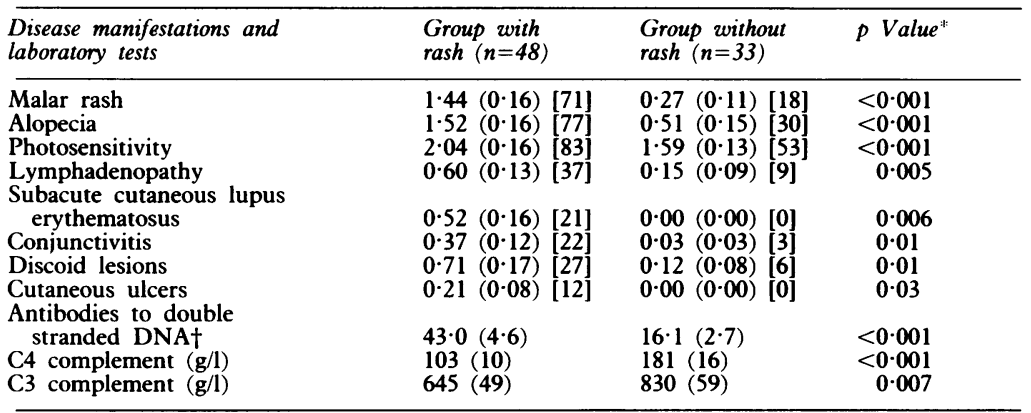

*Wilcoxon's rank sum test.

†Farr method. Range 0-100\%; normal $<20 \%$. origin (43\% $v 50 \%$ Ashkenazi) between the groups with and without rash.

\section{RELATION BETWEEN RASH AND DISEASE MANIFESTATIONS}

A significant difference was observed between the two groups for various disease manifestations and laboratory tests (table 3). As expected, the group with rash had a higher occurrence of various other cutaneous signs such as malar rash, alopecia, photosensitivity, subacute cutaneous lupus lesions, conjunctivitis, discoid lesions, and cutaneous ulcers. The group with rash graded significantly higher for lymphadenopathy and higher levels of antibodies to double stranded DNA and lower complement levels. The peak prednisone dose was significantly higher for the group with rash $(35.5$ $(3.7) v 23.2(4.0) \mathrm{mg} /$ day $(\mathrm{p}=0.03))$, and the group with rash had significantly more patients receiving drugs against malaria $(67 v 20 \%$ $(\mathrm{p}=0.0001))$. No significant difference was observed between the groups for arthritis $(2 \cdot 27$ $(0.4) v 3.06(0.7)(\mathbf{p}=0.7))$, or renal and central nervous system disease. Peak serum creatinine (99.9 (8.8) v 128.2 (33.4) $\mu \mathrm{mol}(\mathrm{p}=0.4))$, blood urea nitrogen, and urine protein excretion did not differ significantly between the groups. Twenty nine per cent of patients with rash had central nervous system disease with convulsions, psychosis, or cerebritis versus $21 \%$ of patients without rash $(p=0 \cdot 6)$.

\section{RELATION BETWEEN RASH AND DISEASE \\ ACTIVITY}

Table 4 gives correlations between the rash at its highest measured value and concomitantly assessed variables. As shown, there was a significant correlation between rash and other cutaneous manifestations such as discoid lesions, subacute cutaneous lupus lesions, malar rash, mouth ulcers, and cutaneous vasculitis. There was no significant correlation between rash and disease activity index $(r=0.09)$ or other signs of disease exacerbation such as fever $(r=0.04)$, or arthritis $(r=-0.05)$. Rash did not correlate significantly with prednisone dose $(\mathrm{r}=0 \cdot 17)$.

\section{RELATION BETWEEN RASH AND OTHER}

CUTANEOUS SIGNS

From all patient examinations, $32 \%$ of patients had a positive recording for rash without a concomitant positive recording for the more specific cutaneous signs.

Table 4 Correlation between rash and other disease manifestations monitored at time of maximum rash according to examination by a doctor

\begin{tabular}{llc}
\hline Disease manifestation & $\begin{array}{l}\text { Correlation } \\
\text { coefficient }\end{array}$ & p Value \\
\hline Discoid lesions & 0.48 & $<0.001$ \\
Subacute cutaneous lupus & 0.39 & $<0.001$ \\
$\quad$ erythematosus & 0.36 & 0.001 \\
Mouth ulcers & 0.34 & 0.002 \\
Malar rash & 0.26 & 0.02 \\
\hline Cutaneous vasculitis & & \\
\hline
\end{tabular}




\section{Discussion}

Much work has been published about the clinical presentations of SLE in general and about the various cutaneous manifestations of the disease. ${ }^{1-3}$ Most workers, however, concentrate on the cutaneous lesions that are specific for SLE such as malar rash or discoid lesions. Dubois reported the various cutaneous manifestations in patients with SLE. ${ }^{6}$ Three subgroups, comprising $35 \%$ of his patients, parallel our patients in the group with rash: maculopapular lesions, non-specific; maculopapular lesions, sun exposed areas (photosensitivity has a separate heading); and urticaria. In addition to being a manifestation of SLE, the rash could have many other causes not directly related to SLE such as being drug induced, due to intercurrent infection, or the manifestation of another specific dermatological disease.

Although not much has been reported about such a rash, two different viewpoints are evident. Rothfield, a rheumatologist, describes nonspecific maculopapular rash as the second most common skin finding. ${ }^{2}$ On the other hand, Callen, a dermatologist, classified SLE cutaneous lesions as lupus erythematosus specific histological pattern and lupus erythematosus nonspecific mucocutaneous lesions.' 'Rash' could possibly fit the second category under the heading 'urticaria'. 6 Only little information is given about 'urticaria', but Dubois mentions that 'the development of urticaria in a patient with systemic lupus erythematosus should lead the physician to carefully evaluate for active systemic disease'. 6

Thus neither viewpoint provides sufficient information-one hardly reports rash and the other reports a high prevalence of rash in lupus patients, but without details about its prevalence or expression. Although the meaning of 'rash' is not well clarified in patient with SLE, it is used in advanced studies of the disease ${ }^{7}$ and three of the recommended systems for assessment of SLE disease activity use 'rash', 89 or 'erythematous, maculopapular rash'10 as a parameter for scoring disease activity.

Our study showed a $59 \%$ prevalence of rash, according to examination by a doctor, and rash was the second most prevalent cutaneous finding secondary to photosensitivity. Patients with rash had, as expected, a significantly higher prevalence of various other cutaneous manifestations. An interesting finding that patients with rash had significantly more lymphadenopathy is as yet unexplained. On the other hand, rash was not more common among patients with renal or central nervous system disease. Patients with rash had more pathological serological tests: higher levels of antibodies to double stranded DNA and lower C3 and C4 complement levels. They were also receiving higher doses of prednisone, and, as expected, used more drugs against malaria. Rash correlated with various other cutaneous lesions but not with disease activity index or other signs of disease exacerbation.
Rash was significantly more common among younger patients. Studies investigating the relation between SLE and age observed no difference between age groups for rash, ${ }^{11}$ though the studies do not refer to 'rash' specifically. In previous studies we did not find any relation betwen age and photosensitivity ${ }^{12}$ or alopecia. ${ }^{13}$

More interaction between rheumatologists and dermatologists will lead to more specific diagnosis of the cutaneous lesions in patients with SLE. We have no doubt that some of our findings of rash could be attributed, by a more dermatologically oriented examination, to one of the more specific cutaneous manifestations of SLE. Thus our report of $32 \%$ of patients with rash and no other skin sign could actually be lower. We report our findings as seen by a rheumatologist and, as such, it is important to understand the significance of rash. Rash is a common finding in patients with SLE. It is related to other cutaneous manifestations, serological markers, and treatment with higher doses of corticosteroids. Patients with rash, however, do not seem to have more severe systemic disease with respect to renal or central nervous system manifestations, and rash is not correlated with disease exacerbation. Thus the existence or appearance of rash, in patients with SLE should not automatically lead to augmentation of systemic treatment. Awareness of these findings by the doctor might lead to a better understanding and management of the disease.

1 Callen J P. Mucocutaneous changes in patients with systemic lupus erythematosus. Rheum Dis Clin North Am 1988; 14 79-97.

2 Rothfield N F. Systemic lupus erythematosus: clinical aspects and treatment. In: McCarty D J, ed. Arthritis and allied conditions. Philadelphia: Lea \& Febiger, 1985: 913-5.

3 Schur P H. Clinical features of SLE. In: Kelley W N, Harri E D Jr, Ruddy S, Sledge C B, eds. Textbook of rheumatology. Philadelphia: Saunders, 1989: 1104-8.

4 Tan E M, Cohen A S, Fries J F, et al. The 1982 revised criteria for the classification of systemic lupus erythematosus. Arthritis Rheum 1982; 25: 1271-7.

5 Symmons D P M, Coppock J S, Bacon P A et al. Development and assessment of a computerized index of Development and assessment of a computerized index of clinical disease activity in systemic lupus
(Appendix 2). Qf Med 1988; 69: 927-37.

6 Dubois E L. The clinical picture of systemic lupus ery thematosus. In: Dubois E L, ed. Lupus erythematosus. Los Angeles: University of Southern California Press, 1974 $294-5$

7 Liang M H, Rogers M, Larson M, et al. The psychosocia impact of systemic lupus erythematosus and rheumatoid arthritis. Arthritis Rheum 1984; 27: 13-19.

8 Morimoto C, Sano H, Abe T, Homma M, Steinberg A D. Correlation between clinical activity of systemic lupus Correlation between the DNA antibody immune complexes. F Immunol 1982; 139: 1960-5.

9 Lockshin M D, Reinitz E, Druzin M L, Murrman M, Estes D. Lupus pregnancy. Am $\mathcal{F}$ Med 1984; 77: 893-8.

10 Liang M H, Socher S A, Larson M G, Schur P H. Reliability and validity of six systems for the clinical assessment of disease activity in systemic lupus erythematosus. Arthritis Rheum 1989; 32: 1107-18.

11 Ward M M, Polisson R P. A meta-analysis of the clinica manifestations of older-onset systemic lupus erythematosus. Arthritis Rheum 1989; 32: 1226-32.

12 Wysenbeek A J, Block D A, Fries J F. Prevalence and expression of photosensitivity in systemic lupus erythematosus. Ann Rheum Dis 1989; 48: 461-3.

13 Wysenbeek A J, Leibovici L, Amit M, Weinberger A. Alopecia in systemic lupus erythematosus. Relation to disease manifestations. $\mathcal{F}$ Rheumatol 1991; 18: 1185-6. 